\title{
Stratification of polymer mixtures in drying droplets: hydrodynamics and diffusion
}

\author{
Michael P. Howard ${ }^{1, a)}$ and Arash Nikoubashman $\left.2, b\right)$ \\ ${ }^{1)}$ McKetta Department of Chemical Engineering, University of Texas at Austin, Austin, TX 78712, \\ United States \\ ${ }^{2)}$ Institute of Physics, Johannes Gutenberg University Mainz, Staudingerweg 7, 55128 Mainz, \\ Germany
}

We study the evaporation-induced stratification of a mixture of short and long polymer chains in a drying droplet using molecular simulations. We systematically investigate the effects of hydrodynamic interactions (HI) on this process by comparing hybrid simulations accounting for HI between polymers through the multiparticle collision dynamics technique with free-draining Langevin dynamics simulations neglecting the same. We find that the dried supraparticle morphologies are homogeneous when HI are included but are stratified in core-shell structures (with the short polymers forming the shell) when HI are neglected. The simulation methodology unambiguously attributes this difference to the treatment of the solvent in the two models. We rationalize the presence (or absence) of stratification by measuring phenomenological multicomponent diffusion coefficients for the polymer mixtures. The diffusion coefficients show the importance of not only solvent backflow but also HI between polymers in controlling the dried supraparticle morphology.

\section{INTRODUCTION}

Understanding microstructure formation during the drying of a volatile solvent from a mixture is essential for engineering coatings, $\frac{11}{1}$ polymer nanocomposites,, 2 and nanocrystal superlattices,,$\underline{3}$ among many technologies. The dried microstructure depends on multiple controllable parameters, including processing conditions like temperature ${ }^{4 / 5}$ relative humidity, $[6$ and solvent properties. ${ }^{7}$ For example, when these conditions cause fast drying, colloidal suspensions densify and crystallize from the solvent-air interface, while under slow drying conditions, the crystal nucleates from the bulk, $\frac{811}{11}$ the quality of the resulting crystal depends on the specific processing pathway ${ }^{5 / 12}$ Multicomponent mixtures exhibit even richer behavior because their constituents can compositionally segregate during drying, 13 holding great promise for assembling functional materials through simple single-step processing.

In particular, the formation of layered films from mixtures of differently sized solutes has received considerable recent attention ${ }^{13}$ Film morphology plays an important role in, e.g., tuning the refractive and reflective characteristics of optical materials, $14 \mid 15$ improving the properties of pressure-sensitive adhesives, 16 and fabricating superhydrophobic coatings. ${ }^{17}$ Experiments and computer simulations showed that well stabilized mixtures of colloidal particles, $\frac{18,[29}{29}$ polymers, ${ }^{30 \mid 31]}$ or both. ${ }^{30 \mid 32}$ will stratify by size during fast drying to form films with a top layer enriched in the smaller component. Similar "small-on-top" stratification also occurs in evaporating droplets, which can be exploited to produce supraparticles with coreshell morphologies $\sqrt[33]{35}$ These supraparticles are promising for applications in catalysis due to their distinctive hi-

\footnotetext{
a)Electronic mail: mphoward@utexas.edu

b) Electronic mail: anikouba@uni-mainz.de
}

erarchical structures ${ }^{36}$ and tunable porosity ${ }^{37}$ or as structural colorants. 38

In order to understand and control the morphology of such materials, different theoretical models were proposed to predict microstructure and stratification during drying! ${ }^{19 / 25 / 39} \sqrt{41}$ Previously, we developed one mode ${ }^{25 / 30}$ using the framework of dynamic density functional theory (DFT) $\stackrel{42}{44}$ This model belongs to a general class of multicomponent diffusion models for stratification. $\underline{40 / 41}$ In experiments, temperature and pressure gradients inside the drying mixture typically relax faster than the solutes diffuse, so the temperature and pressure can be considered approximately constant. The theories then postulate that the diffusive flux $\mathbf{j}_{i}$ of each component $i$ in an $n+1$ component system (comprising $n$ solutes and the solvent) can be generally written as

$$
\mathbf{j}_{i}=\rho_{i}\left(\mathbf{u}_{i}-\overline{\mathbf{u}}\right)=-\sum_{j=0}^{n} L_{i j} \nabla \mu_{j},
$$

where $\rho_{i}$ and $\mathbf{u}_{i}$ are the number density and average velocity of component $i$, respectively, $\overline{\mathbf{u}}$ is the velocity of a chosen reference frame, $L_{i j}$ is a phenomenological Onsager coefficient, and $\mu_{j}$ is the chemical potential of component $j$. The Onsager coefficients must be measured (or assumed) and are symmetric $\left(L_{i j}=L_{j i}\right)$ if Onsager's reciprocal relations hold. ${ }^{45}$ The chemical potential gradients are thermodynamic driving forces for diffusion that are self-generated by the drying process and can be computed based on the solute concentration profiles using, e.g., virial expansions of the free energy ${ }^{41}$ or more accurate free-energy functionals. $\underline{25 \mid 30}$

The reference frame can be simply chosen as the stationary laboratory $(\overline{\mathbf{u}}=\mathbf{0})$, but it is often convenient to define the fluxes relative to a velocity that is a weighted function of $\mathbf{u}_{i} \cdot \frac{46}{6}$ The volume-averaged velocity $\overline{\mathbf{u}}_{\mathrm{v}}=$ $\sum_{i=0}^{n} v_{i} \rho_{i} \mathbf{u}_{i}$ (with $v_{i}$ being the volume of component $i$ ) is particularly expedient if the drying mixture is incompressible because $\overline{\mathbf{u}}_{\mathrm{v}}=\mathbf{0}$ so that there is no net volume 
flux. Making use of the reciprocal relations and that $\sum_{i=0}^{n} v_{i} \mathbf{j}_{i}=\mathbf{0}$ in this reference frame yields

$$
\mathbf{j}_{i}=-\sum_{j=1}^{n} L_{i j} \nabla \mu_{j}^{\prime}
$$

where $\mu_{j}^{\prime}=\mu_{j}-\left(v_{j} / v_{0}\right) \mu_{0}$ is an exchange chemical potential that gives the free-energy change to insert a solute $j$ and remove an equivalent volume of solvent, designated as component 0 . However, the chemical potential gradients are not generally independent of each other because they satisfy the Gibbs-Duhem relationship, $\sum_{i=0}^{n} \rho_{i} \nabla \mu_{i}=0$ at constant temperature and pressure, in local thermodynamic equilibrium. The solvent can then be eliminated from Eq. (1) by redefining

$$
\mathbf{j}_{i}=-\sum_{j=1}^{n} \Lambda_{i j} \nabla \mu_{j},
$$

where we stress that the solvent chemical potential $\mu_{0}$ and flux $\mathbf{j}_{0}$ are no longer considered independent, and new effective coefficients $\Lambda_{i j}$ have been defined from the original $L_{i j}$. Equation (3) can be derived in other reference frames, even in one where all $\mathbf{j}_{i}$ are independent and Eq. (2) does not hold, so we will focus on this effective formulation.

Within such multicomponent diffusion models, smallon-top stratification occurs when the total driving force on the larger solute is sufficiently strong compared to that on the smaller solute so that the two separate as they codiffuse ${ }^{19}$ We and others $25[30141$ effectively assumed dominant diagonal couplings, $\Lambda_{i i} \approx \rho_{i} D_{i} /\left(k_{\mathrm{B}} T\right)$, with negligible off-diagonal couplings, $\Lambda_{i, j \neq i} \approx 0$, where $k_{\mathrm{B}}$ is Boltzmann's constant, $T$ is the temperature, and $D_{i}$ is the equilibrium self-diffusion coefficient of component $i$. When cross-interactions between solutes were included in the chemical potentials, $\frac{41}{1}$ these models predicted stratification qualitatively resembling that observed in experiments ${ }^{\sqrt{19|21| 22}}$ and were even in quantitative agreement with free-draining implicit-solvent computer simulations of drying polymer mixtures $\frac{30}{30}$ However, it was recently demonstrated that these models overpredict the extent of stratification compared to experiments of drying mixtures of colloidal particles. $\frac{13134}{134}$ The overprediction is thought to originate from solvent effects like backflow and/or hydrodynamic coupling that are missing from the models $47 \mid 48$

To remedy this shortcoming, an alternative explanation for stratification was proposed based on the concept of diffusiophoresis. $\underline{48149}$ Sear and Warren analyzed the migration of a single, infinitely large colloid in an ideal polymer solution with hard excluded-volume interactions between the colloid and polymers. ${ }^{48}$ The solvent was taken into account using a continuum description of a thin film flow near the surface of the colloid that is excluded to the polymers. In this picture, the colloid migrates towards regions of lower polymer density with diffusiophoretic velocity

$$
\mathbf{u}_{\mathrm{c}}=-\frac{R_{\mathrm{g}}^{2} k_{\mathrm{B}} T}{2 \eta} \nabla \rho_{\mathrm{p}},
$$

where $R_{\mathrm{g}}$ is the radius of gyration of the polymer, $\eta$ is the viscosity, and the subscripts "c" and "p" denote the colloid and polymers, respectively. Stratification occurs when the large colloid moves down the polymer gradient faster than the polymers themselves. The diffusiophoretic velocity of the colloid can be rewritten as a flux in the form of Eq. (3),

$$
\mathbf{j}_{\mathrm{c}}=-\frac{R_{\mathrm{g}}^{2} \rho_{\mathrm{c}}}{2 \eta} \nabla \rho_{\mathrm{p}} .
$$

This flux might be identified as an off-diagonal coupling, $\Lambda_{\mathrm{cp}}=R_{\mathrm{g}}^{2} \rho_{\mathrm{c}} \rho_{\mathrm{p}} /(2 \eta)$, between the chemical potential gradient of the polymer and the flux of the colloid by assuming $\Lambda_{\mathrm{cc}} \approx 0$ and that the polymer chemical potential is dominated by its own (ideal) contributions, $\nabla \mu_{\mathrm{p}} \approx k_{\mathrm{B}} T \nabla \ln \left(\lambda_{\mathrm{p}}^{3} \rho_{\mathrm{p}}\right)$ where $\lambda_{\mathrm{p}}$ is the thermal wavelength of the polymer. We note, however, that this splitting is not unique because $\nabla \rho_{\mathrm{p}}$ contributes to both $\nabla \mu_{\mathrm{c}}$ for the colloid and $\nabla \mu_{\mathrm{p}}$ for the polymers in nonideal mixtures, and there may be additional terms in $\Lambda_{\mathrm{cc}}$ and $\Lambda_{\mathrm{cp}}$ that effectively cancel each other $\stackrel{48}{ }$

Predictions based on the diffusiophoresis model are in better agreement with experiments than prior diffusion models but are still not quantitatively accurate. $\frac{13}{\text { The }}$ reason for this discrepancy is not obvious, but may be due to some of the approximations that were required to render the calculation of $\mathbf{u}_{\mathrm{c}}$ analytically tractable. For example, solutes have finite size ratios in experiments (typically 10:1 or smaller), but the model assumes that the colloid is much larger than the polymers. Larger solutes like the colloid are also not infinitely dilute in experiments, and so can interact with each other in ways that are not easily accounted for within this model. Generally, most of the mixtures that have been studied experimentally are not sufficiently dilute that they can be assumed to be thermodynamically ideal as in the Asakura-Oosawa treatment of the polymers, $\frac{48}{4}$ particularly for colloidal mixtures and/or once the mixture concentrates during drying. To date, nonidealities like skin-layer formation 150 or jamming $\underline{49}$ have only been taken into account in an ad hoc fashion. Last, the diffusiophoretic picture becomes more complex for solutes that can be penetrated by solvent and/or deform (e.g, polymer mixtures), as this complicates the analysis of the fluid flow! 51

Computer simulations taking into account solvent effects and hydrodynamic interactions (HI) can play a key role in addressing some of these questions. ${ }^{52}$ Simulations can model the solute, size, or concentration regimes that are highly relevant to experiments but are not amenable to a purely theoretical analysis. Simulations also resolve microscopic detail that can be used to stringently test theoretical models and identify key physics, both thermodynamic and hydrodynamic in nature, that are required 
to improve them. For example, chemical potentials, which are challenging to measure in experiments, can be computed directly in simulations using, e.g., Widom's test insertion method! ${ }^{53}$ Different treatments of solventmediated interactions can also be systematically included or excluded from a simulation model in ways that cannot be achieved in experiments. This approach has been used to show the importance of HI in setting the microstructure of drying colloidal suspensions, $\frac{1154}{115}$ but their role in stratification is still debated!29

One of us previously used both explicit-solvent and implicit-solvent molecular simulations to probe the role of HI in the stratification of drying polymer mixtures in a thin film. 31 The polymers were initially dissolved in a solvent explicitly represented as a Lennard-Jones fluid in vapor-liquid coexistence. The explicit solvent not only propagated HI, but also contributed to the effective interactions between the polymers. The implicitsolvent simulations neglected hydrodynamic coupling between polymers, but importantly, both the effective interactions between polymers and their equilibrium selfdiffusion coefficients were matched at infinite dilution. It was found, after extensive testing of the two models, that the presence of $\mathrm{HI}$ effectively suppressed small-on-top stratification. Although ultimately effective, one potential challenge of comparing separate explicit-solvent and implicit-solvent simulation models like these is that thermodynamic and hydrodynamic effects can become convoluted if the model interactions are not perfectly matched and/or if the implicit-solvent model has limited transferability to conditions at which it was not parameterized. Interfacial effects were also handled differently between the two models, ${ }^{[55}$ leaving open questions about their role in setting the microstructure. Therefore, it is preferable to make comparisons like these between simulations using exactly the same effective interactions for the solutes and interfaces so that the only difference between models is the presence or absence of HI.

Given this context, we set two goals for this work. Our first aim was to explore the possibility of stratification of a mixture of short and long polymers in a drying droplet, which has not been previously investigated. We simulated this process for the same coarse-grained polymer model using two different treatments of the solvent and corresponding polymer dynamics: one incorporating HI between polymers and one neglecting the same (Sec. II). Consistent with prior work, $\stackrel{31}{,}$ the mixture stratified to form a core-shell supraparticle morphology when HI were neglected, but did not stratify when HI were included (Sec. IIIB). Our second aim was to rationalize this behavior using a multicomponent diffusion model. We used nonequilibrium simulations to measure the transport coefficients that couple the polymer fluxes to their chemical potential gradients (Sec. IIIC), finding qualitatively different behaviors between the two solvent treatments that were consistent with the presence or absence of stratification. Our study strongly supports the importance of $\mathrm{HI}$ in stratification phenomena, and we advocate in- corporating these interactions in future theoretical and computational models.

\section{MODEL AND METHODS}

The polymers comprising component $i$ were modeled as bead-spring chains of $M_{i}$ spherical beads (monomers), each having diameter $\sigma$ and mass $m$. The interactions between the monomers were purely repulsive and modeled using the Weeks-Chandler-Andersen potential,,$[56$

$$
U_{\mathrm{m}}(r)= \begin{cases}4 \varepsilon\left[\left(\frac{\sigma}{r}\right)^{12}-\left(\frac{\sigma}{r}\right)^{6}\right]+\varepsilon, & r \leq 2^{1 / 6} \sigma \\ 0, & r>2^{1 / 6} \sigma\end{cases}
$$

where $r$ is the center-to-center distance between a pair of monomers and $\varepsilon$ sets the energy scale for the repulsion. Bonded monomers additionally interacted through a finitely extensible nonlinear elastic potential,

$$
U_{\mathrm{b}}(r)= \begin{cases}-\frac{k r_{0}^{2}}{2} \ln \left[1-\frac{r^{2}}{r_{0}^{2}}\right], & r \leq r_{0} \\ \infty, & r>r_{0}\end{cases}
$$

with spring constant $k=30 \varepsilon / \sigma^{2}$ and maximum bond length $r_{0}=1.5 \sigma$ to prevent unphysical chain crossing! 58 These interactions correspond to good solvent conditions for dilute polymer solutions.

A droplet with radius $R$ was created by confining the monomers to a spherical domain using the repulsive part of a harmonic potential,

$$
U_{\mathrm{d}}(\mathbf{r})=\left\{\begin{array}{ll}
0, & |\mathbf{r}| \leq R-\sigma / 2 \\
\varepsilon_{\mathrm{d}}(|\mathbf{r}|-R+\sigma / 2)^{2}, & |\mathbf{r}|>R-\sigma / 2
\end{array},\right.
$$

where $|\mathbf{r}|$ is the distance of a monomer at position $\mathbf{r}$ from the center of the sphere, and $\varepsilon_{\mathrm{d}}$ controls the strength of the repulsion (indirectly, the surface tension). We chose $\varepsilon_{\mathrm{d}}=100 \varepsilon / \sigma^{2}$ to ensure all monomers stayed fully immersed within the droplet. Equation (8) assumes a contact angle of $0^{\circ}$ for the monomers with the interface and neglects any capillary attractions between monomers at the interface. It also enforces a spherical shape at all times, which may impede deformation or buckling during the late stages of drying.

Evaporation was mimicked by reducing the droplet radius as a function of time $t$. For a free liquid droplet, the rate of drying is limited by diffusion of the solvent through the surrounding air, so the time-dependent droplet radius is,, 3460

$$
R^{2}=R_{0}^{2}-\frac{\alpha}{4 \pi} t
$$

where $R_{0}$ is the initial droplet radius and $\alpha$ is the rate of change of surface area that depends on physical properties such as the solvent's vapor-liquid coexistence densities and vapor diffusivity. The corresponding receding 
speed of the interface $v$ is

$$
v=-\frac{\mathrm{d} R}{\mathrm{~d} t}=\frac{\alpha}{8 \pi R} .
$$

This drying model neglects potential decreases in the rate of evaporation due to the formation of a skin layer at the interface 50 However, this approximation should have only a minor impact on our results, as recent experiments of drying binary colloidal droplets found that $\alpha$ was nearly constant until the droplet was completely dried. 34

HI between polymers in the droplet were treated approximately using the multiparticle collision dynamics (MPCD) technique. ${ }^{52[6162]}$ The solvent was modeled explicitly as point particles with mass $m_{0}$ whose motion was governed by alternating streaming and collision steps. In the streaming step, the solvent particles were moved ballistically for a period of time $\Delta t_{0}$. Then, the solvent particles and monomers were sorted into cubic cells with edge length $\sigma$, subject to a random shift to ensure Galilean invariance of the algorithm. ${ }^{63} \mathrm{~A}$ momentumexchanging collision step was then performed between particles in the same cell using the stochastic rotation dynamics (SRD) variant of MPCD, where the velocity of each particle relative to its cell's center-of-mass velocity is rotated by a fixed angle around a randomly oriented axis. ${ }^{61}$ This procedure locally conserves momentum and energy, approximately reproducing HI down to the size of a collision cell $!^{6465}$ Because thermodynamically consistent MPCD algorithms for multiphase systems have only recently been proposed, ${ }^{66}$ we neglected any interactions of the solvent particles with the droplet interface, i.e., the solvent freely flowed through the interface. Hence, the HI were treated as if the polymers were confined to a spherical domain within a bulk fluid, e.g., by a semipermeable membrane. We expect this approximate treatment of the solvent boundary conditions to overpredict solute entrainment ${ }^{67}$ but to be a significant improvement over complete neglect of $\mathrm{HI}$

A droplet having initial radius $R_{0}=50 \sigma$ was filled with $N_{\mathrm{S}}=2440$ short polymers $\left(M_{\mathrm{S}}=10\right)$ and $N_{\mathrm{L}}=305$ long polymers $\left(M_{\mathrm{L}}=80\right)$. Hence, there were $N_{\mathrm{m}}=$ $N_{\mathrm{S}} M_{\mathrm{S}}+N_{\mathrm{L}} M_{\mathrm{L}}=48800$ monomers at an initial monomer volume fraction of $\phi_{\mathrm{m}}=N_{\mathrm{m}} \sigma^{3} /\left(8 R_{0}^{3}\right) \approx 0.05$. Further, the fraction of monomers belonging to long polymers was $\chi=N_{\mathrm{L}} M_{\mathrm{L}} / N_{\mathrm{m}}=0.5$. The spherical droplet was placed at the center of a cubic simulation box with edge length $200 \sigma$, and the entire box was filled with solvent particles at number density $\rho_{0}=5 \sigma^{-3}\left(N_{0}=4 \times 10^{7}\right.$ solvent particles). Periodic boundary conditions were employed for the solvent in all Cartesian directions, which can introduce finite-size artifacts due to coupling of longranged HI between periodic images, $\frac{68}{6 e}$ we chose the box edge length to be twice the droplet diameter, which was the largest size that was computationally feasible for us, to mitigate these effects. The mass of the monomers was set to the average mass of a cell filled with only solvent $\left(m=5 m_{0}\right)$, the SRD collision angle was $130^{\circ}$, and the temperature of the solution was held constant at $T=1.0 \varepsilon / k_{\mathrm{B}}$ using a cell-level Maxwellian thermostat ${ }^{69}$ The solvent collision time was $\Delta t_{0}=0.1 \tau$, with $\tau=$ $\sqrt{m_{0} \sigma^{2} / \varepsilon}$ being the unit of time. The motion of the polymers between stochastic collisions was integrated using a Verlet scheme with time step $\Delta t=0.005 \tau$. All simulations were performed on graphics processing units using HOOMD-blue (version 2.8.1) tended using azplugins (version 0.9.0) ${ }^{73}$ Unless stated otherwise, all simulations were performed with doubleprecision floating-point arithmetic to improve numerical accuracy.

To clearly elucidate the role of HI, we performed implicit-solvent Langevin dynamics (LD) simulations in addition to the MPCD simulations. This approach allows us to use the same polymer model so that any differences between the MPCD and LD simulation results can be traced back to the treatment of the solvent-mediated hydrodynamics. LD simulations model free-draining HI and neglect hydrodynamic coupling between monomers. The polymer self-diffusion coefficient $D_{i}$ in dilute solution then follows Rouse scaling, ${ }^{74 / 75}$

$$
D_{i}=\frac{k_{\mathrm{B}} T}{\xi_{i} M_{i}},
$$

with monomer friction coefficient $\xi_{i}$. In contrast, the MPCD simulations include hydrodynamic coupling, and $D_{i}$ instead follows Zimm scaling, ${ }^{74}[76$

$$
D_{i} \approx \frac{k_{\mathrm{B}} T}{\xi_{i} M_{i}^{\nu}}
$$

where $\nu$ is the fractal dimension of the polymer $(\nu \approx$ 0.588 for linear chains in a good solvent).

To approximately match the long-time diffusive dynamics of the polymers between the MPCD and LD simulations, we followed our previous approach $\frac{11131}{1}$ and adjusted $\xi_{i}$ in the LD simulations so that we obtained the same value of $D_{i}$ in dilute solution as in the MPCD simulations. We first performed MPCD simulations of pure polymer solutions $(\chi=0.0$ or 1.0$)$ in cubic simulation boxes with edge length $80 \sigma$. The monomer volume fraction was set to $\phi_{\mathrm{m}}=0.01$, leading to monomer concentrations $\rho_{\mathrm{m}}$ well below the overlap concentration $\rho_{\mathrm{m}}^{*}$ for both the short and long polymers $\left(\rho_{\mathrm{m}} / \rho_{\mathrm{m}}^{*} \approx 0.04\right.$ and 0.26 , respectively). We computed $D_{i}$ from the mean squared displacement of the polymer centers of mass during simulations of length $10^{5} \tau$. We performed three independent simulations for the short polymers and five independent simulations for the long polymers to improve statistics, finding $D_{\mathrm{S}}=1.08 \times 10^{-2} \sigma^{2} / \tau$ and $D_{\mathrm{L}}=2.7 \times 10^{-3} \sigma^{2} / \tau$ with measurement uncertainties of $0.2 \%$ and $5 \%$, respectively, based on the standard error of the mean. This measurement, combined with Eq. (11), gave $\xi_{\mathrm{S}}=9.3 m_{0} / \tau$ and $\xi_{\mathrm{L}}=4.6 m_{0} / \tau$ for the monomer friction coefficients in the LD simulations. In what follows, we will indicate results from the MPCD simulations (including $\mathrm{HI}$ ) as "+HI", whereas results from the LD simulations (without HI) will be indicated as "-HI". 


\section{RESULTS}

\section{A. Equilibrium properties}

We first confirmed that the $+\mathrm{HI}$ and $-\mathrm{HI}$ simulations gave similar equilibrium properties for bulk polymer solutions. We conducted $+\mathrm{HI}$ and $-\mathrm{HI}$ simulations at various monomer volume fractions $\left(0.05 \leq \phi_{\mathrm{m}} \leq 0.20\right)$ and compositions $(0.0 \leq \chi \leq 1.0)$, using a similar protocol as for fitting $\xi_{i}$ except that we now performed only one simulation per state point. We characterized the conformation of the polymers through the average gyration tensor,

$$
\mathbf{G}=\frac{1}{M} \sum_{i=1}^{M}\left\langle\Delta \mathbf{r}_{i} \Delta \mathbf{r}_{i}^{T}\right\rangle
$$

where $\Delta \mathbf{r}_{i}$ is the vector to monomer $i$ from the polymer center of mass. The radius of gyration was taken as $R_{\mathrm{g}}=\left(G_{x x}+G_{y y}+G_{z z}\right)^{1 / 2}$. As expected, we found that the polymers were isotropic and that the $+\mathrm{HI}$ and -HI simulations produced the same $R_{\mathrm{g}}$. We determined $R_{\mathrm{g}, \mathrm{L}} \approx 6.1 \sigma$ for the long polymers at $\phi_{\mathrm{m}}=0.05$, which decreased to $R_{\mathrm{g}, \mathrm{L}} \approx 5.4 \sigma$ at $\phi_{\mathrm{m}}=0.20$. The short chains shrank less than the long chains, having $R_{\mathrm{g}, \mathrm{S}} \approx 1.7 \sigma$ at $\phi_{\mathrm{m}}=0.05$ and $R_{\mathrm{g}, \mathrm{S}} \approx 1.6 \sigma$ at $\phi_{\mathrm{m}}=0.20$. In all cases, the size of the chains was essentially independent of the composition $\chi$ at constant $\phi_{\mathrm{m}}$.

Figure 11 compares the bulk self-diffusion coefficients of the short $\left(D_{\mathrm{S}}\right)$ and long $\left(D_{\mathrm{L}}\right)$ polymers as a function of $\phi_{\mathrm{m}}$ and $\chi$ for the $+\mathrm{HI}$ and $-\mathrm{HI}$ simulations. In both cases, $D_{\mathrm{S}}$ and $D_{\mathrm{L}}$ decreased with increasing $\phi_{\mathrm{m}}$, as expected from the concomitant increase in solution viscosity. Interestingly, changing the composition $\chi$ had a much weaker effect at fixed $\phi_{\mathrm{m}}$. For the short chains, $D_{\mathrm{S}}$ agreed well between the $+\mathrm{HI}$ and $-\mathrm{HI}$ simulations for the short-polymer solutions $(\chi=0.0)$, and the agreement generally improved with increasing $\phi_{\mathrm{m}}$, where HI are increasingly screened. Deviations between the $+\mathrm{HI}$ and $-\mathrm{HI}$ results became more pronounced as the fraction of long chains $\chi$ increased, but the overall agreement was good (maximum deviation of 25\%) for all simulated compositions. For the long chains, the $+\mathrm{HI}$ and $-\mathrm{HI}$ simulations matched well at high $\phi_{\mathrm{m}}$, and the agreement was better when the mixture had fewer short chains (larger $\chi)$. Despite differences at some compositions, the overall agreement between the $+\mathrm{HI}$ and $-\mathrm{HI}$ data was again reasonable (maximum deviation of $35 \%$ ).

\section{B. Evaporation}

Having established the equilibrium properties of the polymer solutions and good consistency between the $+\mathrm{HI}$ and -HI models, we then performed nonequilibrium simulations of the solutions in drying droplets. The evaporation process is typically characterized by the droplet Péclet numbers, $\mathrm{Pe}_{i}$, which describe the relative contributions of advection and diffusion to the motion of the

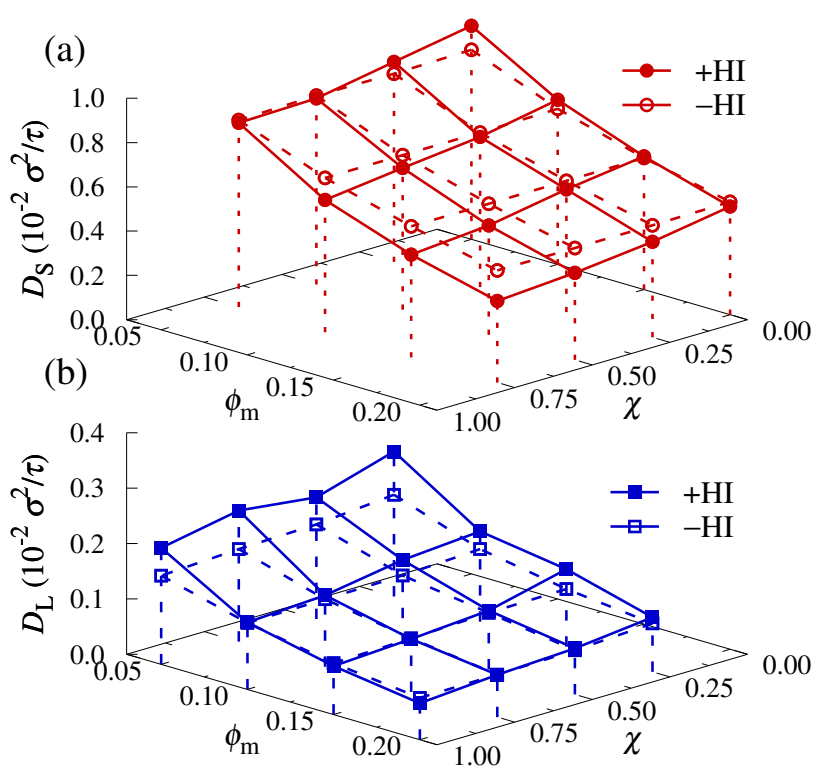

FIG. 1. Self-diffusion coefficients for the (a) short polymers $D_{\mathrm{S}}$ (red circles) and (b) long polymers $D_{\mathrm{L}}$ (blue squares) in bulk solutions as a function of monomer volume fraction $\phi_{\mathrm{m}}$ and composition $\chi$ with HI (filled symbols) and without HI (open symbols).

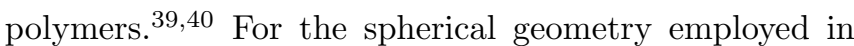
this work, we defined $\mathrm{Pe}_{i}=v_{0} R_{0} / D_{i}$ using the initial droplet radius $R_{0}$ as the characteristic length scale and the initial evaporation speed $v_{0}=\alpha /\left(8 \pi R_{0}\right)$ as the characteristic velocity. Note that $\mathrm{Pe}_{\mathrm{S}}$ and $\mathrm{Pe}_{\mathrm{L}}$ differ for the short and long polymers due to their different diffusion coefficients and $\mathrm{Pe}_{\mathrm{L}}>\mathrm{Pe}_{\mathrm{S}}$. We conducted simulations at six drying speeds (Table I) and repeated each simulation eight times for both the $+\mathrm{HI}$ and $-\mathrm{HI}$ models to improve statistics. For computational efficiency, the $+\mathrm{HI}$ drying simulations used mixed-precision arithmetic ${ }^{72}$ and the HI simulations used single-precision arithmetic.

TABLE I. Initial evaporation speed $v_{0}$ (with corresponding rate of change of surface area $\alpha$ ) and Péclet numbers for the short polymers $\mathrm{Pe}_{\mathrm{S}}$ and long polymers $\mathrm{Pe}_{\mathrm{L}}$ in a droplet with initial radius $R_{0}=50 \sigma$.

\begin{tabular}{cccc}
\hline$v_{0}(\sigma / \tau)$ & $\alpha\left(\sigma^{2} / \tau\right)$ & $\mathrm{Pe}_{\mathrm{S}}$ & $\mathrm{Pe}_{\mathrm{L}}$ \\
\hline 0.0001 & 0.13 & 0.46 & 1.8 \\
0.0005 & 0.63 & 2.3 & 9.2 \\
0.001 & 1.3 & 4.6 & 18 \\
0.002 & 2.5 & 9.3 & 37 \\
0.005 & 6.3 & 23 & 92 \\
0.01 & 13 & 46 & 180 \\
\hline
\end{tabular}

Figure 2 shows snapshots of the drying droplet from one of the $+\mathrm{HI}$ and $-\mathrm{HI}$ simulations for $v_{0}=0.002 \sigma / \tau$. These snapshots reveal that the mixture formed a weakly stratified core-shell structure in the -HI simulations, with the shorter polymers enhanced near the droplet-air 
(a)

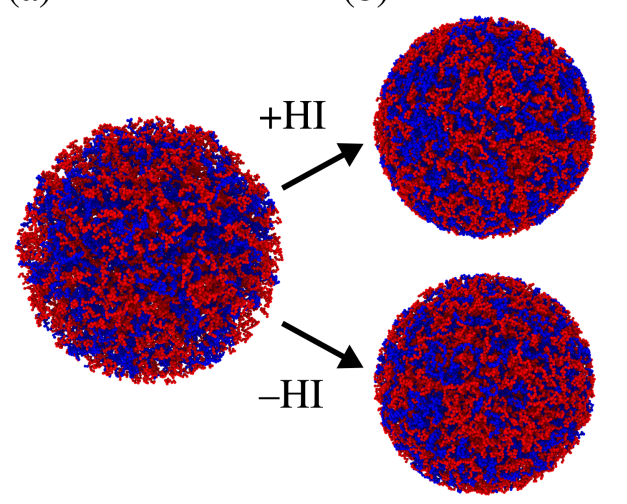

(c)

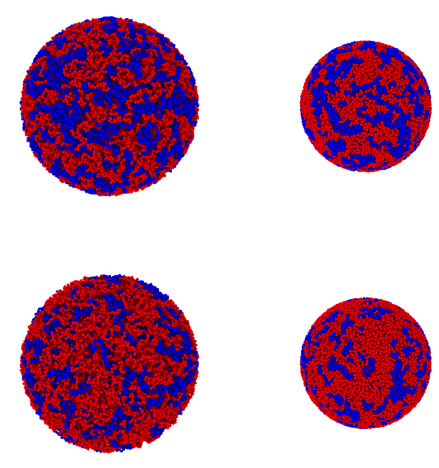

FIG. 2. Simulation snapshots showing the outside of the drying droplet at (a) $R=50.0 \sigma$, (b) $41.8 \sigma$, (c) $33.9 \sigma$, and (d) $25.0 \sigma$ in the $+\mathrm{HI}$ (top) and $-\mathrm{HI}$ (bottom) simulations. The initial evaporation speed was $v_{0}=0.002 \sigma / \tau$. The short and long polymers are colored red and blue, respectively.

interface. It is, however, difficult to visually judge the extent of stratification from these snapshots due to the interpenetration of the polymers. To better assess the presence of stratification, we computed the corresponding average radial density profiles of monomers from the short and long polymers $\rho_{\mathrm{m}, i}$. Figure 3 shows these profiles for the same drying rate and time points as in Fig. 2, averaged over the eight simulations. The profiles confirm the formation of a core-shell morphology in the - HI simulations, but the morphology in the $+\mathrm{HI}$ simulations remained essentially homogeneous. To verify that the stratified structures in the - HI simulations were a result of the drying, we continued the simulations with the final droplet radius held constant at $R=25 \sigma$, and indeed observed that the monomer distributions relaxed to (almost) uniform distributions (dotted lines in Fig. 33).

In order to quantify the degree of stratification near the droplet surface, we computed the average difference in the densities of monomers from the short and long chains in a thin shell comparable to the size of the large polymer,

$$
\Delta \rho_{\mathrm{m}}=\frac{3}{R^{3}-R^{\prime 3}} \int_{R^{\prime}}^{R}\left[\rho_{\mathrm{m}, \mathrm{S}}(r)-\rho_{\mathrm{m}, \mathrm{L}}(r)\right] r^{2} \mathrm{~d} r
$$

where $R^{\prime}=R-2 R_{\mathrm{g}, \mathrm{L}}$ and $R_{\mathrm{g}, \mathrm{L}} \approx 6.1 \sigma$ is the radius of gyration of the long chains at the initial monomer volume fraction $\phi_{\mathrm{m}}=0.05$. The case $\Delta \rho_{\mathrm{m}}>0$ indicates an excess of monomers from short polymers, whereas $\Delta \rho_{\mathrm{m}}<0$ indicates the opposite. In a perfectly homogeneous solution at composition $\chi=0.5$, we expect $\Delta \rho_{\mathrm{m}}=0$. However, even in equilibrium, there is a small (but noticeable) excess of short chains close to the droplet-air interface due to entropic effects (Fig. 3). To remove this inherent offset, we computed $\Delta \rho_{\mathrm{m} \text {,eq }}$ at the final droplet radius $(R=25 \sigma)$ after the mixture equilibrated and subtracted it from $\Delta \rho_{\mathrm{m}}$. Figure 4 shows the resulting order parameter $\Delta \Delta \rho_{\mathrm{m}}=\Delta \rho_{\mathrm{m}}-\Delta \rho_{\mathrm{m}, \text { eq }}$ as a function of initial evaporation speed $v_{0}$ for both the $+\mathrm{HI}$ and $-\mathrm{HI}$ simulations.
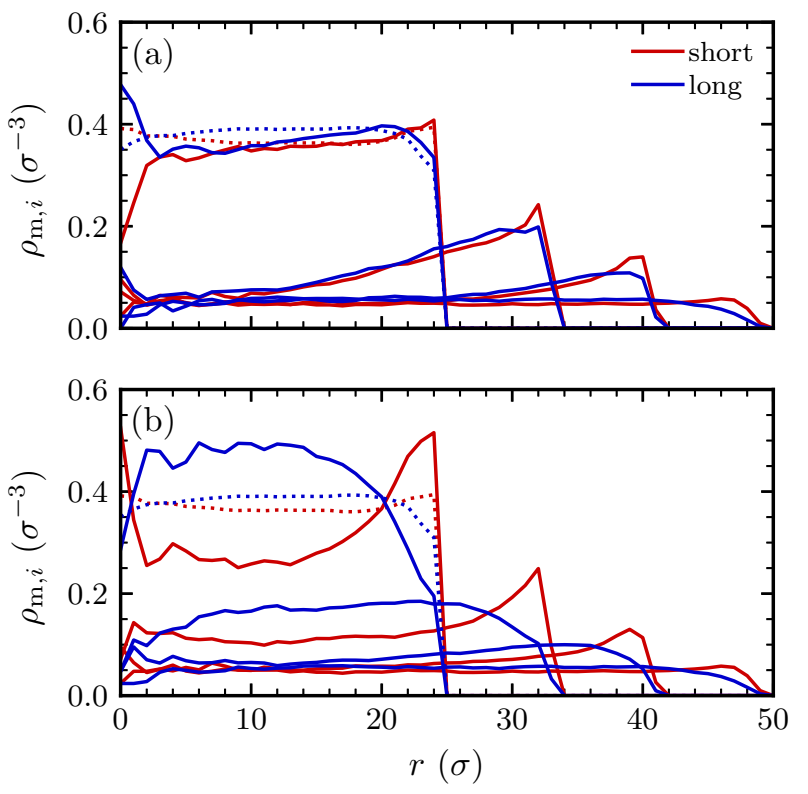

FIG. 3. Radial monomer density profiles $\rho_{\mathrm{m}, i}$ of the short polymers (red) and long polymers (blue) from the (a) $+\mathrm{HI}$ and (b) $-\mathrm{HI}$ simulations for the same $v_{0}$ and $R$ as in Fig. 2 The equilibrium profiles for $R=25.0 \sigma$ are also shown as dotted lines.

These data clearly show that the polymer mixtures remained essentially homogeneous in the $+\mathrm{HI}$ simulations for all investigated evaporation speeds. In contrast, there was distinct stratification in the -HI simulations, which became more pronounced as the droplets dried faster.

Despite these differences in overall morphology, we found that the conformations of the polymers within the droplet were essentially the same in both the $+\mathrm{HI}$ and -HI simulations. We computed the gyration tensor of the polymers in the droplet before drying and at the end of drying using Eq. 133 and determined radial 


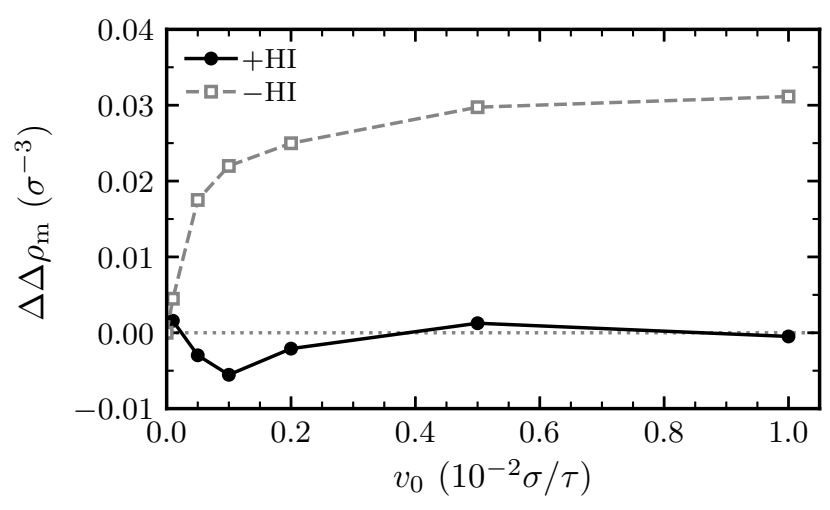

FIG. 4. Order parameter $\Delta \Delta \rho_{\mathrm{m}}$ as a function of initial evaporation speed $v_{0}$ for the $+\mathrm{HI}$ and $-\mathrm{HI}$ simulations. $\Delta \Delta \rho_{\mathrm{m}}>0$ indicates small-on-top stratification, while $\Delta \Delta \rho_{\mathrm{m}}<0$ indicates more long polymers near the surface compared to the equilibrium distribution.

profiles by averaging according to the position of the polymer centers of mass. We then computed the normal component $R_{\mathrm{g}}^{(\mathrm{n})}$ and tangential component $R_{\mathrm{g}}^{(\mathrm{t})}$ of the total radius of gyration $R_{\mathrm{g}}$ relative to the droplet surface. Figure 5 shows these components for the $+\mathrm{HI}$ simulations normalized relative to their values in bulk at the initial volume fraction and composition $\left(\phi_{\mathrm{m}}=0.05\right.$ and $\chi=0.5$ ). Before drying, both the long and short polymers had isotropic shapes $\left(R_{\mathrm{g}}^{(\mathrm{n})}=R_{\mathrm{g}}^{(\mathrm{t})} / \sqrt{2}\right)$ except near the surface of the droplet, where both tended to be slightly stretched tangential to the surface and compressed normal to the surface. The long polymers were affected over a longer distance from the surface, as expected; these effects are fully consistent with equilibrium depletion of the long polymer near the surface (Fig. 33). Both polymers shrank as the mixture concentrated during drying, with the increased density affecting the long polymers more than the short polymers (as in the equilibrium bulk solutions, see Section III A). However, both components of $R_{\mathrm{g}}$ for the short polymers stayed close to their equilibrium profiles (i.e., those obtained when we stopped the drying simulations at $R=25.0 \sigma$ and relaxed the mixture, dotted line), while the long polymers tended to be slightly smaller after drying compared to equilibrium. Although Fig. 5 shows profiles for the $+\mathrm{HI}$ simulations, the $-\mathrm{HI}$ simulations had nearly identical final profiles for the components of $R_{\mathrm{g}}$. This may be partially due to the fact that the total monomer densities are similar in both simulations even though the local polymer compositions are drastically different (Fig. 33. The most striking differences in structure were obtained in the (mesoscopic) droplet morphology rather than the (microscopic) polymer conformations, with HI between polymers seemingly playing an important role in setting the droplet morphology.
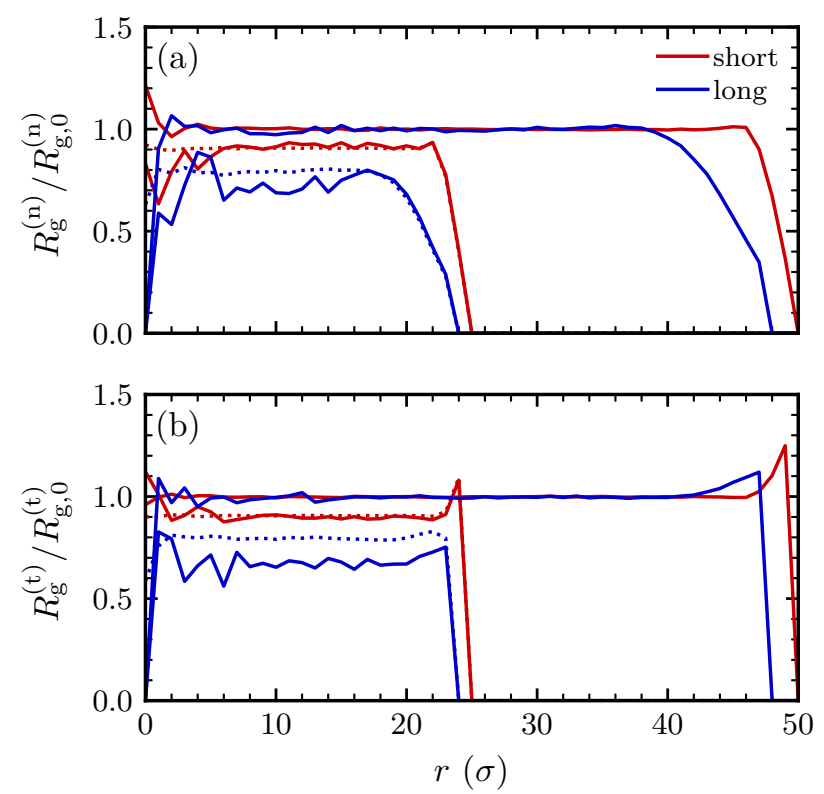

FIG. 5. Radial profiles of the (a) normal component $R_{\mathrm{g}}^{(\mathrm{n})}$ and (b) tangential component $R_{\mathrm{g}}^{(\mathrm{t})}$ of the radius of gyration for the short polymers (red) and long polymers (blue) before $(R=50.0 \sigma)$ and after $(R=25.0 \sigma)$ drying at $v_{0}=0.002 \sigma / \tau$ in the + HI simulations. The values are normalized by their bulk values at the initial volume fraction and composition $\left(\phi_{\mathrm{m}}=0.05\right.$ and $\left.\chi=0.5\right)$, i.e., $R_{\mathrm{g}, 0}^{(\mathrm{n})}=R_{\mathrm{g}, 0} / \sqrt{3}$ and $R_{\mathrm{g}, 0}^{(\mathrm{t})}=$ $R_{\mathrm{g}, 0} \sqrt{2 / 3}$ with $R_{\mathrm{g}, 0}$ being the bulk value of the radius of gyration discussed in Section III A The equilibrium profiles for $R=25.0 \sigma$ are also shown as dotted lines. Profiles after drying in the $-\mathrm{HI}$ simulations were essentially the same and have been omitted for clarity.

\section{Onsager coefficients}

The qualitatively different morphologies obtained in the $+\mathrm{HI}$ and $-\mathrm{HI}$ drying simulations are completely consistent with prior simulations that compared stratification of polymer solutions in drying films with and without $\mathrm{HI} \cdot{ }^{[11}$ It is then clearly important to include HI, which are inherent to experiments, to build predictive models for this dynamic process. However, this task can be computationally demanding even with a mesoscale simulation technique like MPCD. Continuum multicomponent diffusion models (Eq. (1)) remain attractive in this respect, but the solvent must be properly accounted for in the thermodynamic model through the chemical potentials $\mu_{i}$ and in the transport model through the phenomenological Onsager coefficients $L_{i j}$. Unlike prior work, our polymer model has identical thermodynamics in both the $+\mathrm{HI}$ and $-\mathrm{HI}$ simulations, so the polymer chemical potentials $\mu_{\mathrm{S}}$ and $\mu_{\mathrm{L}}$ are the same in both. Comparison of the two simulations then allows us to isolate the role of the solvent in the process and how HI impact $L_{i j}$.

To facilitate this comparison, it will be preferable to convert the Onsager coefficients $L_{i j}$ defined by Eq. (1) 
to effective phenomenological coefficients $\Lambda_{i j}$ defined by Eq. (3). This conversion is necessary because the + HI simulations model a true three-component mixture, whereas the -HI simulations model an effective twocomponent mixture. In the $-\mathrm{HI}$ simulations, the implicit solvent is a stationary background whose properties do not depend on the polymers, so it is trivially the case that $\Lambda_{i j}=L_{i j}$. In the +HI simulations, the motion of the solvent is coupled to the motion of the polymers because linear momentum is conserved locally so that a net force on (and flux of) the polymers implies a counterforce on (and backflow of) the solvent in the opposite direction. As a result, the mass-averaged velocity,

$$
\overline{\mathbf{u}}_{\mathrm{m}}=\frac{\sum_{i=0}^{n} m_{i} \rho_{i} \mathbf{u}_{i}}{\sum_{i=0}^{n} m_{i} \rho_{i}}
$$

(with $m_{i}$ being the mass of component $i$ ), is zero if the net force on the mixture is zero, making $\overline{\mathbf{u}}_{\mathrm{m}}$ an appropriate reference velocity for defining $\mathbf{j}_{i}$ in the $+\mathrm{HI}$ simulations. We will assume that the Gibbs-Duhem relationship between the chemical potentials at constant temperature and pressure is satisfied in the $+\mathrm{HI}$ simulations as a result of the local momentum conservation and zero net force, and also that the Onsager reciprocal relations hold. With these assumptions and using $\overline{\mathbf{u}}_{\mathrm{m}}$ in Eq. (1), the effective phenomenological coefficients are obtained as

$$
\Lambda_{i j}=\sum_{k=1}^{n} L_{i k}\left(\delta_{k j}+\frac{m_{k} \rho_{j}}{m_{0} \rho_{0}}\right)
$$

with $\delta_{k j}$ being the Kronecker delta. This relationship fully eliminates the solvent from the phenomenological model. With Eq. (3), the coefficients $\Lambda_{i j}$ allow for direct comparison of how the polymer fluxes depend on gradients of the polymer chemical potentials between the $+\mathrm{HI}$ and $-\mathrm{HI}$ simulations.

We aimed to use simulations to clarify how HI contribute to $\Lambda_{i j}$ and to test assumptions made in prior models. In particular, the coefficient $\Lambda_{\mathrm{LS}}$ that couples the flux of the long polymers to the chemical potential gradient of the short polymers contributes to diffusiophoretic mod-

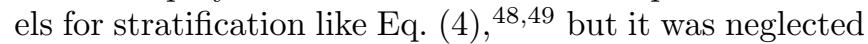
in models derived from dynamic DFT, 25|30|41] where $\Lambda_{\mathrm{LL}}$ was assumed to be dominant. It is unclear which coefficients are required to properly capture the dynamics in concentrated solutions of deformable polymers, for which it is not straightforward to compute the diffusiophoretic coefficient 51

To this end, we measured $\Lambda_{i j}$ directly in bulk polymer mixtures using nonequilibrium simulations ${ }^{77}$ The initial configurations were taken from the equilibrium simulations (Sec. III A). We applied a constant force $\mathbf{F}_{j}=F_{j} \hat{\mathbf{x}}$ to either the short polymers or the long polymers, where $\hat{\mathbf{x}}$ is a unit vector pointing along the $x$ direction. (In practice, this was achieved by distributing an equal force $\left(F_{j} / M_{j}\right) \hat{\mathbf{x}}$ to each bead in the chain.) The constant force plays the role of a linear chemical potential gradient, $\nabla \mu_{j}=-\mathbf{F}_{j}$, without requiring a concentration gradient. In the $+\mathrm{HI}$ simulations, a counterforce
$\mathbf{F}_{0}=-\left(N_{j} / N_{0}\right) \mathbf{F}_{j}$ was applied to the solvent particles to ensure that the entire system was force-free, so there were effectively both polymer and solvent chemical potential gradients in opposite directions. Simulations were run at multiple values of $F_{\mathrm{S}} \leq 0.1 \varepsilon / \sigma$ and $F_{\mathrm{L}} \leq 0.4 \varepsilon / \sigma$ that were sufficiently small that the polymers did not deform significantly ( $G_{x x}$ extended less than $3 \%$ relative to its equilibrium value). The composition was fixed at $\chi=0.5$ and the monomer volume fraction was varied in the range $0.05 \leq \phi_{\mathrm{m}} \leq 0.20$. The coefficients $\Lambda_{i j}$ were determined by fitting the average velocity $\mathbf{u}_{i}$ of component $i$ in a stationary reference frame $(\overline{\mathbf{u}}=\mathbf{0})$ according to Eq. (3),

$$
\rho_{i} \mathbf{u}_{i}=\Lambda_{i j} \mathbf{F}_{j} .
$$

We measured the average velocities every $2.5 \tau$ during a simulation of length $10^{5} \tau$, and we discarded the first $10 \%$ of the data to allow the system to achieve a steady flux. We computed the average velocities using the remainder of the data and estimated uncertainties from the standard error between 5 subdivided blocks of the data.

In the $+\mathrm{HI}$ simulations, the net force on the polymer mixture is zero and linear momentum is conserved, so the reference frame for measuring $\mathbf{u}_{i}$ can be considered both the laboratory frame and the mass-averaged frame given by $\overline{\mathbf{u}}_{\mathrm{m}}$ (Eq. 15p). We confirmed that $\overline{\mathbf{u}}_{\mathrm{m}} \lesssim 10^{-8} \sigma / \tau$ for all investigated cases, as expected. In a pure polymer solution, momentum conservation implies that a polymer flux in one direction must be opposed by a solvent backflow. (An analogous backflow occurs in colloidal suspensions, which are usually assumed to be incompressible, or to have no net volume flux, so a flux of colloids implies a solvent backflow.) In a two-polymer mixture with solvent, a flux of one of the polymer species can be balanced by not only the solvent backflow but also potentially a flux of the other polymer species. The distribution of these fluxes will depend on the interactions between polymers and the coefficients $\Lambda_{i j}$. We emphasize that the mixtures remained homogeneous in both the $+\mathrm{HI}$ and $-\mathrm{HI}$ simulations, and that there was no net flow in the $+\mathrm{HI}$ simulations, supporting the assumption of constant pressure in our analysis of the $+\mathrm{HI}$ simulations.

In the -HI simulations, there is no explicit solvent, and $\mathbf{F}_{j}$ imposes a net force. The polymers still reached a steady-state velocity because the implicit solvent was dissipative, but unlike the $+\mathrm{HI}$ simulations, there was no explicit backflow of solvent (i.e., $\mathbf{u}_{0}=\mathbf{0}$ ). The force on the polymers in the $-\mathrm{HI}$ simulations implies a balancing force on the implicit solvent to make the mixture forcefree. This effect cannot be easily included in the inhomogeneous drying simulations, $\frac{19|21| 25|26| 30}{\text { but }}$ it can be approximately treated in the bulk measurements of $\Lambda_{i j}$. Assuming a force-free polymer-implicit-solvent mixture and total momentum conservation, as in the $+\mathrm{HI}$ simulations, the measured flux of the polymers implies a solvent backflow,

$$
\mathbf{u}_{0}=-\frac{m}{m_{0} \rho_{0}}\left(M_{\mathrm{S}} \rho_{\mathrm{S}} \mathbf{u}_{\mathrm{S}}+M_{\mathrm{L}} \rho_{\mathrm{L}} \mathbf{u}_{\mathrm{L}}\right) .
$$


The - HI simulations can then be regarded as having been conducted in either the stationary laboratory frame or the frame that moves with the solvent at velocity $\mathbf{u}_{0}$. Velocities measured in the latter moving frame can be shifted into a stationary one where the solvent flows and $\overline{\mathbf{u}}_{\mathrm{m}}=\mathbf{0}$ using Eq. 18). To make complete comparison with the $+\mathrm{HI}$ simulations, we will present results for $\Lambda_{i j}$ from the $-\mathrm{HI}$ simulations taking $\mathbf{u}_{i}$ both unshifted (as in the evaporation simulations) and shifted to account for backflow. Even with shifting, though, the polymer fluxes and implied solvent backflow in the -HI simulations can still differ from the $+\mathrm{HI}$ simulations, as these may depend on the presence of $\mathrm{HI}$.

Figure 6 shows one example of the measured average velocities $u_{x, i}=\mathbf{u}_{i} \cdot \hat{\mathbf{x}}$ as a function of $F_{\mathrm{S}}$ for $\phi_{\mathrm{m}}=0.10$. The relationship between $u_{x, i}$ and $F_{\mathrm{S}}$ was linear, giving another indication that $F_{\mathrm{S}}$ was sufficiently small to reliably perform the measurement. In both the $+\mathrm{HI}$ and -HI simulations, the short polymers migrated in the direction of the applied force, as expected. In the $+\mathrm{HI}$ simulations (Fig. 6a), we measured not only a solvent backflow $\left(u_{x, 0}<0\right)$ but also a counterflow of the long polymers $\left(u_{x, \mathrm{~L}}<0\right)$. In contrast, in the $-\mathrm{HI}$ simulations (Fig. 6b), both the short polymers and long polymers migrated in the same direction $\left(u_{x, \mathrm{~L}}>0\right)$ in the laboratory frame. Shifting to account for the solvent backflow using Eq. (18) (Fig. 6p, dashed lines) did not qualitatively alter this behavior but did decrease both $u_{x, \mathrm{~S}}$ and $u_{x, \mathrm{~L}}$. Analogous behavior was observed in the simulations having force $F_{\mathrm{L}}$ applied to the long polymers with the role of the short and long polymers exchanged. However, larger $F_{\mathrm{L}}$ than $F_{\mathrm{S}}$ was required to obtain comparable $u_{x, i}$ due to the decreased mobility of the long polymers.

We extracted all four $\Lambda_{i j}$ from simulations with either $F_{\mathrm{S}}$ or $F_{\mathrm{L}}$ applied using Eq. (17), shown in Fig. 7. We first considered the diagonal coefficients $\Lambda_{\mathrm{SS}}$ (Fig. 7 a) and $\Lambda_{\mathrm{LL}}$ (Fig. $7 \mathrm{~d}$ ) coupling the flux of the polymers to their own chemical potential gradients. We found that $\Lambda_{\mathrm{SS}}$ and $\Lambda_{\mathrm{LL}}$ were both positive and consistently smaller with HI than without HI, indicating that HI retard the motion of the polymers as in Batchelor's analysis of sedimenting colloidal suspensions. ${ }^{78}$ This effect was (relatively) more significant for the long polymers (Fig. 7d) than for the short polymers (Fig. 77) at low $\phi_{\mathrm{m}}$. The measured $\Lambda_{i i}$ are in modest agreement with the ansatz in the dynamic DFT models, $\Lambda_{i i} / \rho_{i} \approx D_{i} / k_{\mathrm{B}} T$, with an error of roughly $50 \%$ or less for both the $+\mathrm{HI}$ and $-\mathrm{HI}$ simulations. However, more significant qualitative differences between the $+\mathrm{HI}$ and $-\mathrm{HI}$ simulations are apparent for the off-diagonal coefficients $\Lambda_{\mathrm{LS}}$ (Fig. $7 \mathrm{~b}$ ) and $\Lambda_{\mathrm{SL}}$ (Fig. 7k), which have different signs in the $+\mathrm{HI}$ and $-\mathrm{HI}$ simulations. The off-diagonal contributions are positive and promote diffusion without $\mathrm{HI}$ but are negative and inhibit diffusion with HI. (We also computed the underlying off-diagonal Onsager coefficients $L_{\mathrm{LS}}$ and $L_{\mathrm{SL}}$ by inverting Eq. (16); they showed the same qualitative differences as $\Lambda_{\mathrm{LS}}$ and $\Lambda_{\mathrm{SL}}$ and are symmetric, as expected (Fig. 8).) Inclusion of solvent backflow in the -HI simu-
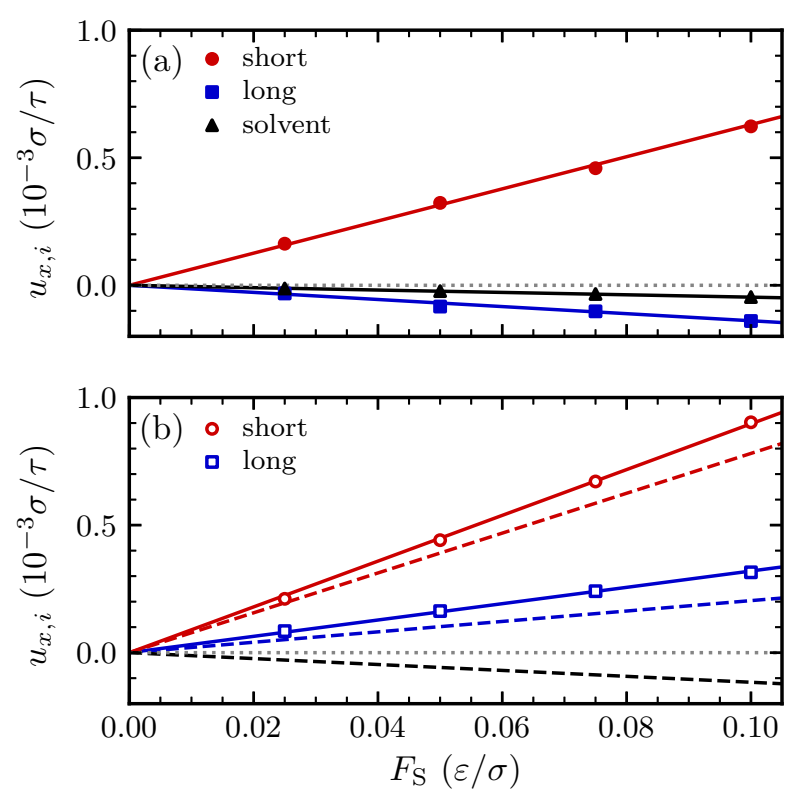

FIG. 6. Average velocity $u_{x, i}$ of the short (red circles) and long (blue squares) polymers when the short polymers are dragged with a constant force $F_{\mathrm{S}}$ in the (a) $+\mathrm{HI}$ (filled symbols) and (b) - HI (open symbols) simulations at $\phi_{\mathrm{m}}=0.10$ and $\chi=0.5$. In (a), the measured average solvent velocity is also shown (black triangles). In (b), the implied solvent velocity was computed using Eq. (18) and is shown as a black dashed line. The polymer velocities in this reference frame are also shown as dashed lines. The estimated measurement uncertainty is smaller than the symbol size.

lations quantitatively shifted all $\Lambda_{i j}$; however, the same qualitative differences were apparent in the off-diagonal components. Evidently, not only solvent backflow but also HI between polymers play an important role in setting $\Lambda_{i j}$ that needs to be considered.

We focus on $\Lambda_{\mathrm{LS}}$, which contributes to the diffusiophoretic coefficient for the long polymers. For all tested $\phi_{\mathrm{m}}, \Lambda_{\mathrm{LS}}>0$ in the $-\mathrm{HI}$ simulations, but $\Lambda_{\mathrm{LS}}<0$ in the $+\mathrm{HI}$ simulations. If the diagonal contribution $\Lambda_{\mathrm{LL}}$ to the long-polymer flux is neglected, the change in sign of $\Lambda_{\mathrm{LS}}$ fully explains the presence or absence of stratification in the $-\mathrm{HI}$ and $+\mathrm{HI}$ simulations, respectively. However, the picture is more complex for nondilute polymer solutions. First, the short-polymer concentration profile (chemical potential gradient) evolves dynamically and is determined by both the short-polymer and longpolymer concentrations through $\Lambda_{\mathrm{SS}}$ and $\Lambda_{\mathrm{SL}}$ (Fig. 7F). Hence, small quantitative differences in all $\Lambda_{i j}$ between the $+\mathrm{HI}$ and $-\mathrm{HI}$ simulations may contribute to the final morphology. Second, the flux of the long polymers due to their own chemical potential is nonnegligible because neither the short polymers nor the long polymers are dilute. This, in particular, may promote stratification when $\Lambda_{\mathrm{LL}}>0$ even as $\Lambda_{\mathrm{LS}}<0$ tends to suppress it in the + HI simulations, and the net effect of these two contributions will depend on the magnitudes of $\nabla \mu_{\mathrm{S}}$ and 

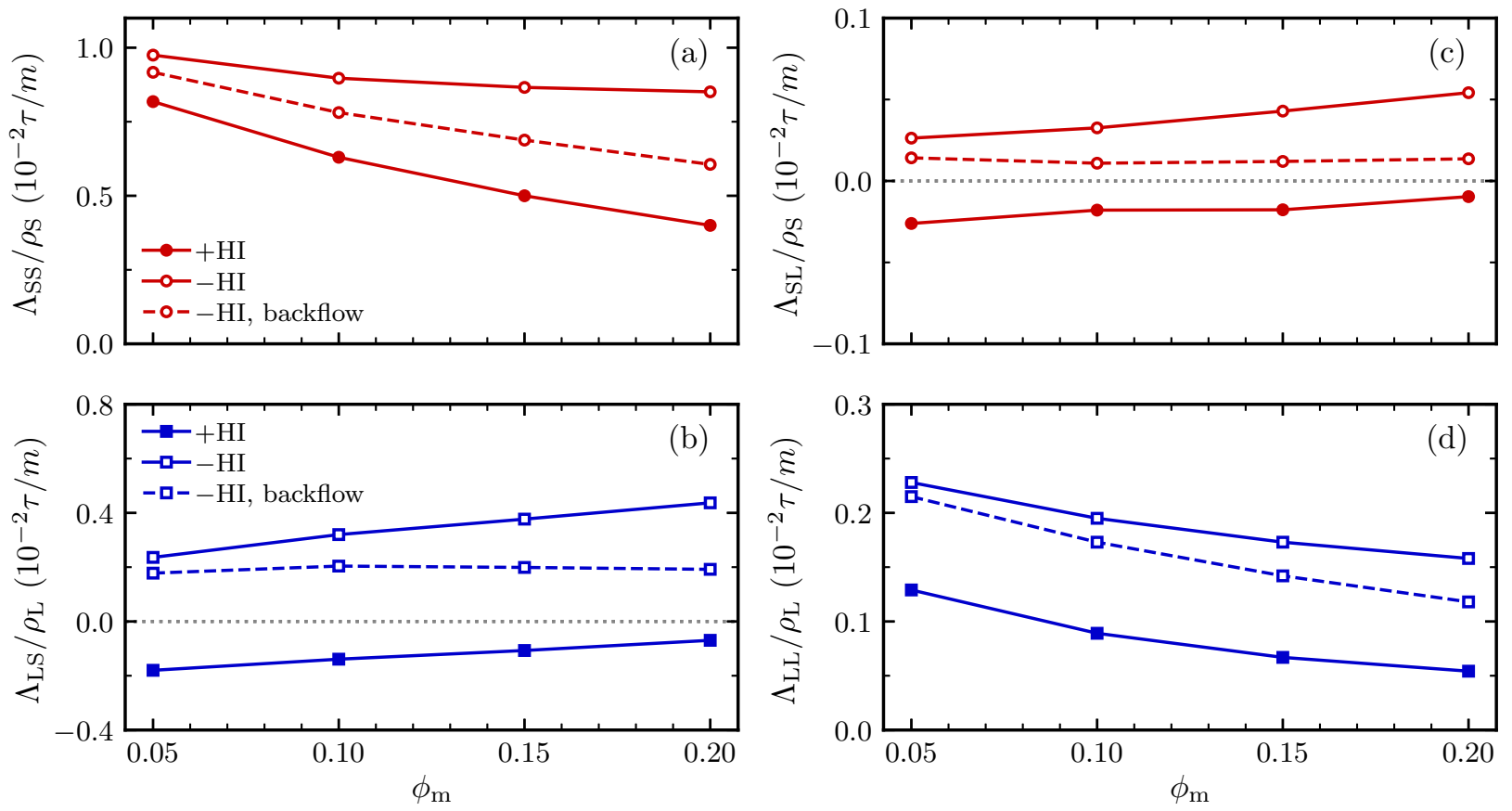

FIG. 7. Effective Onsager coefficients (a) $\Lambda_{\mathrm{SS}}$, (b) $\Lambda_{\mathrm{LS}}$, (c) $\Lambda_{\mathrm{SL}}$, and (d) $\Lambda_{\mathrm{LL}}$ as functions of monomer volume fraction $\phi_{\mathrm{m}}$ at fixed composition $\chi=0.5$ for the $+\mathrm{HI}$ (filled symbols) and $-\mathrm{HI}$ simulations (open symbols). (Note the different scales for $\Lambda_{i j}$ in each panel.) In the -HI simulations, the coefficients are shown for both the reference frame where the solvent is stationary (solid lines) and the reference frame where the polymer velocities are shifted to account for solvent backflow (dashed lines) using Eq. 18. As in Fig. 6, the red circles (top row) indicate coefficients for the flux of the short polymers, while the blue squares (bottom row) indicate coefficients for the flux of the long polymers. The estimated uncertainties from fitting the simulation data to Eq. (17) are smaller than the symbol size.

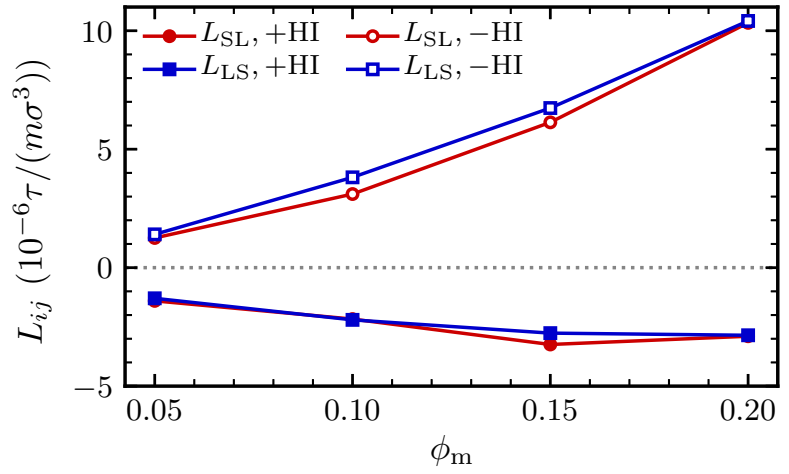

FIG. 8. Off-diagonal Onsager coefficients $L_{\mathrm{SL}}$ (red circles) and $L_{\mathrm{LS}}$ (blue squares) as functions of monomer volume fraction $\phi_{\mathrm{m}}$ at fixed composition $\chi=0.5$, corresponding to the simulations in Fig. 77 The coefficients for the +HI simulations (filled symbols) were obtained by inverting Eq. (16), while the coefficients for the $-\mathrm{HI}$ simulations (open symbols) are trivially $L_{i j}=\Lambda_{i j}$.

$\nabla \mu_{\mathrm{L}}$.

For the bead-spring polymer model studied, the polymer chemical potentials can be well approximated using a hard-chain equation of state ${ }^{30179 / 80}$ We separate the chemical potential into ideal and excess parts, $\nabla \mu_{j}=$ $\nabla \mu_{j}^{\text {id }}+\nabla \mu_{j}^{\text {ex }}$. The ideal term $\nabla \mu_{j}^{\text {id }}=k_{\mathrm{B}} T \nabla \ln \left(\lambda_{j}^{3} \rho_{j}\right)$ depends on the individual density of component $j$, while $\nabla \mu_{j}^{\text {ex }}$ is proportional to $M_{j}$ and depends on the total monomer volume fraction $\phi_{\mathrm{m}}$ and polymer number density $\rho_{\mathrm{S}}+\rho_{\mathrm{L}}$. In a sufficiently dense solution, the ideal contribution can be neglected and $\nabla \mu_{j} \approx \nabla \mu_{j}^{\text {ex }}$ to first approximation, so $\nabla \mu_{\mathrm{L}} \approx\left(M_{\mathrm{L}} / M_{\mathrm{S}}\right) \nabla \mu_{\mathrm{S}}$. In this regime, the flux of the short or long polymers can then be approximately computed using a single effective coefficient, $\mathbf{j}_{i} \approx \tilde{\Lambda}_{i} \nabla \mu_{\mathrm{S}}$, where

$$
\tilde{\Lambda}_{i}=\Lambda_{i \mathrm{~S}}+\left(\frac{M_{\mathrm{L}}}{M_{\mathrm{S}}}\right) \Lambda_{i \mathrm{~L}}
$$

Small-on-top stratification is usually expected when $\left|\mathbf{u}_{\mathrm{L}}\right|>\left|\mathbf{u}_{\mathrm{S}}\right|$ so that the long polymers separate from the short polymers as they codiffuse, $1925|26| 30 \mid 41$ and therefore an approximate condition for stratification in the nondilute regime is $\tilde{\Lambda}_{\mathrm{L}} / \rho_{\mathrm{L}}>\tilde{\Lambda}_{\mathrm{S}} / \rho_{\mathrm{S}}$ across a range of compositions.

Figure 9 shows $\tilde{\Lambda}_{i} / \rho_{i}$ for both the + HI simulations and the $-\mathrm{HI}$ simulations in the laboratory frame used for the evaporation simulations. In the $-\mathrm{HI}$ simulations, $\tilde{\Lambda}_{\mathrm{L}} / \rho_{\mathrm{L}}>\tilde{\Lambda}_{\mathrm{S}} / \rho_{\mathrm{S}}$ for all $\phi_{\mathrm{m}}$, consistent with the presence of stratification in that model. In contrast, $\tilde{\Lambda}_{\mathrm{L}} / \rho_{\mathrm{L}} \approx \tilde{\Lambda}_{\mathrm{S}} / \rho_{\mathrm{S}}$ in the $+\mathrm{HI}$ simulations for all $\phi_{\mathrm{m}}$, and $\tilde{\Lambda}_{\mathrm{L}} / \rho_{\mathrm{L}}$ and $\tilde{\Lambda}_{\mathrm{S}} / \rho_{\mathrm{S}}$ are both smaller than in the $-\mathrm{HI}$ simulations, suggesting 


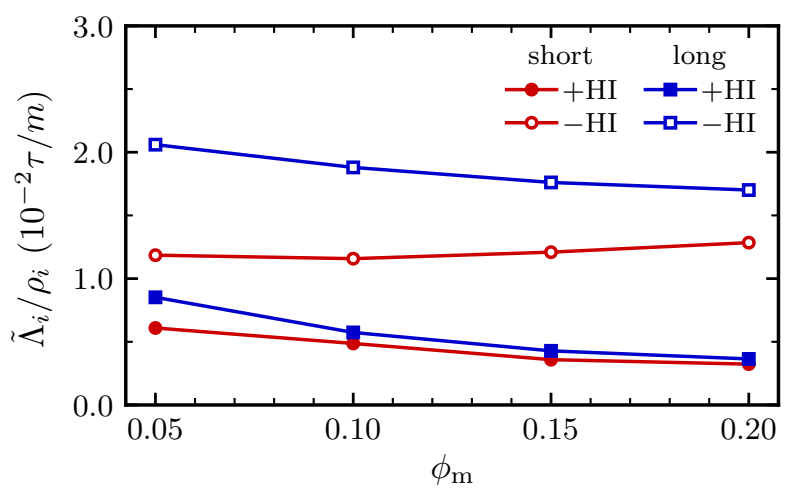

FIG. 9. Approximate coefficients $\tilde{\Lambda}_{i} / \rho_{i}$ for the short (red circles) and long (blue squares) polymers in the $+\mathrm{HI}$ (filled symbols) and - HI (open symbols) simulations, computed from Eq. 19.

that the two components will not readily stratify. Inspection of the relative magnitudes of the diagonal and off-diagonal contributions to $\tilde{\Lambda}_{i}$ (Fig. 7) indicates that the diagonal contributions are in fact more significant. Hence, although there are obvious qualitative differences in the off-diagonal contributions to the diffusive flux in the $+\mathrm{HI}$ and $-\mathrm{HI}$ simulations, the data suggest that it is important to include the diagonal contributions to the flux when modeling stratification in nondilute polymer mixtures, and HI modify the values of these coefficients.

\section{CONCLUSIONS}

We have investigated the microstructures of drying droplets containing mixtures of short and long polymers using computer simulations. By employing models with the same treatment of the polymers but different treatments of the solvent, we have focused in particular on the role of hydrodynamic interactions (HI) between polymers in setting the morphology of the dried supraparticle. In qualitative agreement with prior studies of polymer mixtures in drying thin films, we found that the polymers formed a core-shell morphology (with the short polymers being enriched in the shell) in the simulations without HI, and this stratification became more pronounced with increased evaporation speed. However, the morphology remained homogeneous when HI were included.

We rationalized this behavior using a phenomenological multicomponent diffusion model that connects the diffusive flux of the polymers to gradients in their chemical potentials through effective Onsager coefficients. We measured these coefficients directly in bulk polymer mixtures using nonequilibrium simulations, finding that $\mathrm{HI}$ qualitatively altered off-diagonal coefficients such as the one coupling the flux of long polymers to the chemical potential gradient of the short polymers. However, we found that the diagonal coefficients also played an important role in nondilute mixtures due to the relative magnitudes of the chemical potential gradients. Indeed, the model predicted diffusive fluxes consistent with the presence (or absence) of stratification in the drying simulations when taking all coefficients into account in combination with approximate expressions for the polymer chemical potentials.

Our simulations suggest several promising directions for improving models for evaporation-induced stratification. First, although we were able to rationalize the morphologies in our drying simulations through effective Onsager coefficients, measuring these coefficients can be cumbersome. It will be important to develop theoretical models or constitutive relations that robustly predict Onsager coefficients for different types of polymers or mixtures. For example, mixtures of polymers with chemical incompatibilities may give rise to interesting morphologies, but their Onsager coefficients likely differ from those of the polymers in good-solvent conditions that we studied 51 Second, although we found that there were quantitative differences in the effective Onsager coefficients when solvent backflow was accounted for in simulations without HI, these coefficients were still qualitatively different from those in the simulations with HI. This discrepancy suggests that it is essential to incorporate not only solvent backflow but also hydrodynamic coupling between solutes in simulations and theoretical models of stratification. Last, we focused in this work on the role of HI in the stratification of polymer mixtures. It has been suggested that HI play a qualitatively similar role in the stratification of drying colloidal mixtures; however, there may be quantitative differences due to fundamental hydrodynamic differences between colloids and polymers. A recent study attempted to address this question but used explicit-solvent and implicit-solvent simulation models with interactions that were imperfectly matched, $\stackrel{29}{, 2}$ making it difficult to unambiguously identify the role of HI. A methodology similar to the one we used in this work should help to clarify this question, although a different model for HI is likely required.

\section{AUTHOR CONTRIBUTIONS}

Michael P. Howard: Conceptualization, Methodology, Software, Formal analysis, Writing, Visualization. Arash Nikoubashman: Conceptualization, Methodology, Investigation, Resources, Writing, Funding acquisition.

\section{ACKNOWLEDGMENTS}

We thank the organizers and participants of the CECAM workshop "Applications of Diffusiophoresis in Drying, Freezing, and Flowing Colloidal Suspensions" for stimulating discussions and hospitality during the time in which this work was initiated and Antonia Statt for providing helpful comments on this manuscript. $\mathrm{MPH}$ 
acknowledges support from the Center for Materials for Water and Energy Systems, an Energy Frontier Research Center funded by the U.S. Department of Energy, Office of Science, Basic Energy Sciences under Award \#de-sc0019272. AN acknowledges funding through the German Research Foundation (DFG) under projects NI 1487/2-1 and GRK2516 (\#405552959). Computing time was granted on the supercomputer Mogon at Johannes Gutenberg University Mainz (www.hpc.uni-mainz.de).

\section{DATA AVAILABILITY}

The data that support the findings of this study are available from the authors upon reasonable request.

${ }^{1}$ A. F. Routh, Rep. Prog. Phys. 76, 046603 (2013).

${ }^{2}$ S. K. Kumar, V. Ganesan, and R. A. Riggleman, J. Chem. Phys. 147, 020901 (2017).

${ }^{3}$ M. A. Boles, M. Engel, and D. V. Talapin, Chem. Rev. 116, 11220 (2016).

${ }^{4}$ Y.-H. Ye, F. LeBlanc, A. Haché, and V.-V. Truong, Appl. Phys. Lett. 78, 52 (2001).

${ }^{5}$ S. H. Im and O. O. Park, Langmuir 18, 9642 (2002).

${ }^{6}$ Y.-W. Chung, I.-C. Leu, J.-H. Lee, and M.-H. Hon, Langmuir 22, 6454 (2006).

${ }^{7}$ A. Courty, J. Richardi, P.-A. Albouy, and M.-P. Pileni, Chem. Mater. 23, 4186 (2011).

${ }^{8}$ S. Narayanan, J. Wang, and X.-M. Lin, Phys. Rev. Lett. 93, 135503 (2004).

${ }^{9}$ T. P. Bigioni, X.-M. Lin, T. T. Nguyen, E. I. Corwin, T. A. Witten, and H. M. Jaeger, Nat. Mater. 5, 265 (2006).

${ }^{10}$ M. Wang and J. F. Brady, Soft Matter 13, 8156 (2017).

${ }^{11}$ M. P. Howard, W. F. Reinhart, T. Sanyal, M. S. Shell, A. Nikoubashman, and A. Z. Panagiotopoulos, J. Chem. Phys. 149, 094901 (2018).

${ }^{12}$ S. Cheng and G. S. Grest, J. Chem. Phys. 138, 064701 (2013).

${ }^{13}$ M. Schulz and J. L. Keddie, Soft Matter 14, 6181 (2018)

${ }^{14}$ S. Chhajed, M. F. Schubert, J. K. Kim, and E. F. Schubert, Appl. Phys. Lett. 93, 251108 (2008).

${ }^{15}$ I. Lee, J. Y. Park, S. Gim, K. Kim, S.-H. Cho, C. S. Choi, S.-Y. Song, and J.-L. Lee, ACS Appl. Mater. Interfaces 8, 3326 (2016).

${ }^{16}$ C. Carelli, F. Déplace, L. Boissonnet, and C. Creton, J. Adhes. 83, 491 (2007).

${ }^{17}$ A. B. López, J. C. de la Cal, and J. M. Asua, Polymer 124, 12 (2017).

${ }^{18}$ R. E. Trueman, E. L. Domingues, S. N. Emmett, M. W. Murray, J. L. Keddie, and A. F. Routh, Langmuir 28, 3420 (2012).

${ }^{19}$ A. Fortini, I. Martín-Fabiani, J. L. De La Haye, P.-Y. Dugas, M. Lansalot, F. D'Agosto, E. Bourgeat-Lami, J. L. Keddie, and R. P. Sear, Phys. Rev. Lett. 116, 118301 (2016).

${ }^{20}$ I. Martín-Fabiani, A. Fortini, J. Lesage de la Haye, M. L. Koh, S. E. Taylor, E. Bourgeat-Lami, M. Lansalot, F. D'Agosto, R. P. Sear, and J. L. Keddie, ACS Appl. Mater. Interfaces 8, 34755 (2016).

${ }^{21}$ D. K. Makepeace, A. Fortini, A. Markov, P. Locatelli, C. Lindsay, S. Moorhouse, R. Lind, R. P. Sear, and J. L. Keddie, Soft Matter 13, 6969 (2017).

${ }^{22}$ X. Liu, W. Liu, A. J. Carr, D. Santiago Vazquez, D. Nykypanchuk, P. W. Majewski, A. F. Routh, and S. R. Bhatia, J. Colloid Interface Sci. 515, 70 (2018).

${ }^{23}$ A. J. Carr, W. Liu, K. G. Yager, A. F. Routh, and S. R. Bhatia, ACS Appl. Nano Mater. 1, 4211 (2018).

${ }^{24}$ W. Liu, A. J. Carr, K. G. Yager, A. F. Routh, and S. R. Bhatia, J. Colloid Interface Sci. 538, 209 (2019).

${ }^{25}$ M. P. Howard, A. Nikoubashman, and A. Z. Panagiotopoulos, Langmuir 33, 3685 (2017).
${ }^{26}$ A. Fortini and R. P. Sear, Langmuir 33, 4796 (2017).

${ }^{27}$ Y. Tang, G. S. Grest, and S. Cheng, Langmuir 34, 7161 (2018).

${ }^{28}$ Y. Tang, G. S. Grest, and S. Cheng, Langmuir 35, 4296 (2019).

${ }^{29}$ Y. Tang, G. S. Grest, and S. Cheng, J. Chem. Phys. 150, 224901 (2019).

${ }^{30}$ M. P. Howard, A. Nikoubashman, and A. Z. Panagiotopoulos, Langmuir 33, 11390 (2017).

${ }^{31}$ A. Statt, M. P. Howard, and A. Z. Panagiotopoulos, J. Chem. Phys. 149, 024902 (2018).

${ }^{32}$ S. Cheng and G. S. Grest, ACS Macro Lett. 5, 694 (2016).

${ }^{33}$ L. T. Raju, S. Chakraborty, B. Pathak, and S. Basu, Langmuir 34, 5323 (2018).

${ }^{34}$ W. Liu, J. Midya, M. Kappl, H.-J. Butt, and A. Nikoubashman, ACS Nano 13, 4972 (2019).

${ }^{35}$ T. E. Gartner III, C. M. Heil, and A. Jayaraman, Mol. Syst. Des. Eng. 5, 864 (2020).

${ }^{36}$ K. Hou, J. Han, and Z. Tang, ACS Mater. Lett. 2, 95 (2020).

${ }^{37}$ W. Liu, M. Kappl, and H.-J. Butt, ACS Nano 13, 13949 (2019).

${ }^{38}$ M. Xiao, Z. Hu, T. E. Gartner, III, X. Yang, W. Li, A. Jayaraman, N. C. Gianneschi, M. D. Shawkey, and A. Dhinojwala, Sci. Adv. 5, eaax1254 (2019).

${ }^{39}$ A. F. Routh and W. B. Zimmerman, Chem. Eng. Sci. 59, 2961 (2004).

${ }^{40}$ R. E. Trueman, E. L. Domingues, S. N. Emmett, M. W. Murray, and A. F. Routh, J. Colloid Interface Sci. 377, 207 (2012).

${ }^{41}$ J. Zhou, Y. Jiang, and M. Doi, Phys. Rev. Lett. 118, 108002 (2017).

${ }^{42}$ U. M. B. Marconi and P. Tarazona, J. Chem. Phys. 110, 8032 (1999).

${ }^{43}$ A. J. Archer and R. Evans, J. Chem. Phys. 121, 4246 (2004).

${ }^{44}$ A. J. Archer, J. Phys.: Condens. Matter 17, 1405 (2005).

${ }^{45}$ L. Onsager, Phys. Rev. 37, 405 (1931).

${ }^{46}$ J. B. Brady, Am. J. Sci. 275, 954 (1975).

${ }^{47}$ J. F. Brady, J. Fluid Mech. 667, 216 (2011).

${ }^{48}$ R. P. Sear and P. B. Warren, Phys. Rev. E 96, 062602 (2017).

${ }^{49}$ R. P. Sear, J. Chem. Phys. 148, 134909 (2018).

${ }^{50}$ T. Okuzono, K. Ozawa, and M. Doi, Phys. Rev. Lett. 97, 136103 (2006).

${ }^{51}$ S. Ramírez-Hinestrosa, H. Yoshida, L. Bocquet, and D. Frenkel, J. Chem. Phys. 152, 164901 (2020).

${ }^{52}$ M. P. Howard, A. Nikoubashman, and J. C. Palmer, Curr. Opin. Chem. Eng. 23, 34 (2019).

${ }^{53}$ B. Widom, J. Chem. Phys. 39, 2808 (1963).

${ }_{55}^{54}$ B. Chun, T. Yoo, and H. W. Jung, Soft Matter 16, 523 (2020).

${ }^{55}$ Y. Tang and S. Cheng, Phys. Rev. E 98, 032802 (2018).

${ }^{56}$ J. D. Weeks, D. Chandler, and H. C. Andersen, J. Chem. Phys. 54, 5237 (1971).

${ }^{57}$ M. Bishop, M. H. Kalos, and H. L. Frisch, J. Chem. Phys. 70, 1299 (1979).

${ }^{58}$ K. Kremer and G. S. Grest, J. Chem. Phys. 92, 5057 (1990).

${ }^{59}$ P. Pieranski, Phys. Rev. Lett. 45, 569 (1980).

${ }^{60}$ I. Langmuir, Phys. Rev. 12, 368 (1918).

${ }^{61}$ A. Malevanets and R. Kapral, J. Chem. Phys. 110, 8605 (1999).

${ }^{62}$ G. Gompper, T. Ihle, D. M. Kroll, and R. G. Winkler, in $A d-$ vanced Computer Simulation Approaches for Soft Matter Sciences III Advances in Polymer Science, Vol. 221, edited by C. Holm and K. Kremer (Springer, Berlin, 2009) pp. 1-87.

${ }^{63}$ T. Ihle and D. M. Kroll, Phys. Rev. E 63, 020201(R) (2001).

${ }^{64}$ C.-C. Huang, G. Gompper, and R. G. Winkler, Phys. Rev. E 86, 056711 (2012).

${ }^{65}$ V. Dahirel, X. Zhao, B. Couet, G. Batôt, and M. Jardat, Phys. Rev. E 98, 053301 (2018).

${ }^{66}$ T. Eisenstecken, R. Hornung, R. G. Winkler, and G. Gompper, Europhys. Lett. 121, 24003 (2018).

${ }^{67}$ C. Aponte-Rivera and R. N. Zia, Phys. Rev. Fluids 1, 023301 (2016).

${ }^{68}$ I. C. Yeh and G. Hummer, J. Phys. Chem. B 108, 15873 (2004).

${ }^{69}$ C.-C. Huang, A. Chatterji, G. Sutmann, G. Gompper, and R. G. Winkler, J. Comput. Phys. 229, 168 (2010). 
${ }^{70}$ J. A. Anderson, C. D. Lorenz, and A. Travesset, J. Comput. Phys. 227, 5342 (2008).

${ }^{71}$ J. Glaser, T. D. Nguyen, J. A. Anderson, P. Lui, F. Spiga, J. A. Millan, D. C. Morse, and S. C. Glotzer, Comput. Phys. Commun. 192, 97 (2015).

${ }^{72}$ M. P. Howard, A. Z. Panagiotopoulos, and A. Nikoubashman, Comput. Phys. Commun. 230, 10 (2018).

${ }^{73} \mathrm{https}$ //github.com/mphowardlab/azplugins.

${ }^{74}$ M. Doi and S. F. Edwards, The Theory of Polymer Dynamics (Oxford University Press, New York, 1986).
${ }^{75}$ I. Teraoka, Polymer Solutions: An Introduction to Physical Properties (Wiley, New York, 2002).

${ }^{76}$ K. Mussawisade, M. Ripoll, R. G. Winkler, and G. Gompper, J. Chem. Phys. 123, 144905 (2005).

${ }^{77}$ E. J. Maginn, A. T. Bell, and D. N. Theodorou, J. Phys. Chem. 97, 4173 (1993).

${ }^{78}$ G. K. Batchelor, J. Fluid Mech. 74, 1 (1976).

${ }^{79}$ G. Jackson, W. G. Chapman, and K. E. Gubbins, Mol. Phys. 65, 1 (1988).

${ }^{80}$ W. G. Chapman, G. Jackson, and K. E. Gubbins, Mol. Phys. 65, 1057 (1988). 